\title{
Selective neuromodulation and mutual inhibition within the CA3-CA2 system can prioritize sequences for replay
}

\author{
Tristan M. Stöber ${ }^{1,2,3}$ ( ) | Andrew B. Lehr ${ }^{1,2,4}$ | Torkel Hafting ${ }^{2,5}$ | Arvind Kumar ${ }^{6}$ | \\ Marianne Fyhn ${ }^{2,7}$
}

${ }^{1}$ Department of Computational Physiology, Simula Research Laboratory, Lysaker, Norway

${ }^{2}$ Centre for Integrative Neuroplasticity, University of Oslo, Oslo, Norway

${ }^{3}$ Department of Informatics, University of Oslo, Oslo, Norway

${ }^{4}$ Department of Computational Neuroscience, University of Göttingen, Göttingen, Germany

${ }^{5}$ Institute of Basic Medical Sciences, University of Oslo, Oslo, Norway

${ }^{6}$ Department of Computational Science and Technology, KTH Royal Institute of Technology, Stockholm, Sweden

${ }^{7}$ Department of Biosciences, University of Oslo, Oslo, Norway

\section{Correspondence}

Tristan M. Stöber, Department of Computational Physiology, Simula Research Laboratory, Lysaker, Norway.

Email: tristan.stoeber@posteo.net

Andrew B. Lehr, Department of Computational Neuroscience, University of Göttingen, Göttingen, Germany.

Email: andrew.lehr@phys.uni-goettingen.de

Marianne Fyhn, Centre for Integrative Neuroplasticity, University of Oslo, Oslo, Norway.

Email: marianne.fyhn@ibv.uio.no

Funding information

Research Council of Norway, Grant/Award

Numbers: 248828, 250259; Simula Research Laboratory; University of Oslo; Swedish

Research Council: StratNeuro

\begin{abstract}
To make optimal use of previous experiences, important neural activity sequences must be prioritized during hippocampal replay. Integrating insights about the interplay between CA3 and CA2, we propose a conceptual framework that allows the two regions to control which sequences are reactivated. We suggest that neuromodulatory-gated plasticity and mutual inhibition enable discrete assembly sequences in both regions to support each other while suppressing competing sequences. This perspective provides a coherent interpretation for a variety of seemingly disconnected functional properties of CA2 and paves the way for a more general understanding of CA2.
\end{abstract}

\section{KEYWORDS}

CA2, CA3, consolidation, hippocampus, sequence prioritization

\section{1 | INTRODUCTION}

Tristan M. Stöber and Andrew B. Lehr are equal first authors. Arvind Kumar and Marianne Fyhn are equal senior authors.

To understand the crucial role of the hippocampus for episodic memory, most research has focused on the dentate gyrus (DG) as well as on cornu ammonis subfields 1 and 3 (CA1/CA3). For the most part,

This is an open access article under the terms of the Creative Commons Attribution License, which permits use, distribution and reproduction in any medium, 
hippocampal region CA2 has been considered a transition zone and ignored in the conceptual understanding of the hippocampus. However, in recent years hippocampal region CA2 has received increased attention. Several experimental studies established that CA2 and its distinct neuromodulation are crucial for social recognition memory (DeVito et al., 2009; Meira et al., 2018; Okuyama, Kitamura, Roy, Itohara, \& Tonegawa, 2016; Smith, Avram, Cymerblit-Sabba, Song, \& Young, 2016; Stevenson \& Caldwell, 2014; Wersinger et al., 2004; Wersinger, Caldwell, Christiansen, \& Young, 2007; Wersinger, Ginns, O'carroll, Lolait, \& Young lii, 2002; Wersinger, Temple, Caldwell, \& Young 3rd, 2008; Young, Li, Wersinger, \& Palkovits, 2006). Moreover, experimental data suggest that CA2 plays an important role in several nonsocial behaviors and in controlling hippocampal network dynamics. For example, it appears that CA2 may be involved in temporal sequence memory (DeVito et al., 2009), sharp wave ripples (Alexander et al., 2018; Oliva, Fernández-Ruiz, Buzsáki, \& Berényi, 2016a), CA3 spike timing and place field arrangement (Boehringer et al., 2017), as well as generation of low-gamma oscillations and low-gamma coherence between hippocampus and prefrontal cortex (Alexander et al., 2018). From influencing network dynamics to supporting learning and memory, CA2's role appears diverse. How can we understand such diverse functions of an otherwise small subregion of the hippocampus?

To elucidate CA2's functional role we need to understand the computations it can potentially perform. Such an approach has a long history when studying the function of hippocampal subregions. Based on David Marr's "from-structure-to-function" approach, it has been suggested that CA3 may act as an auto-associative memory unit (Marr, 1971; Papp, Witter, \& Treves, 2007; Rolls, Treves, \& Rolls, 1998). Similarly, the DG, at a computational level, is considered a pattern separator (Gluck \& Rumelhart, 1990; Leutgeb, Leutgeb, Moser, \& Moser, 2007; Treves \& Rolls, 1992). Despite their simplicity, such abstractions of CA3 and DG have provided a powerful conceptual framework to design new experiments exploring the functions of the hippocampus. In this article, we synthesize experimental data about the network architecture and synaptic plasticity in CA2. We propose that at the computational level, CA2 interacts with CA3 to prioritize selected neuronal activity sequences for replay based on contextual and behavioral states. This computational abstraction helps us understand how CA2 can have an important role in a multitude of behaviors beyond social memory. Finally, based on this framework we propose new experiments that can expose the contribution of CA2 in prioritizing neuronal activity sequences.

\section{2 | INPUT AND RECURRENT CONNECTIVITY OF CA2}

In order to elucidate CA2's function it is helpful to zoom out and look at its position within the hippocampus (see Figure 1, upper left panel). CA2 receives direct excitatory input from CA3 (Li, Somogyi, Ylinen, \&

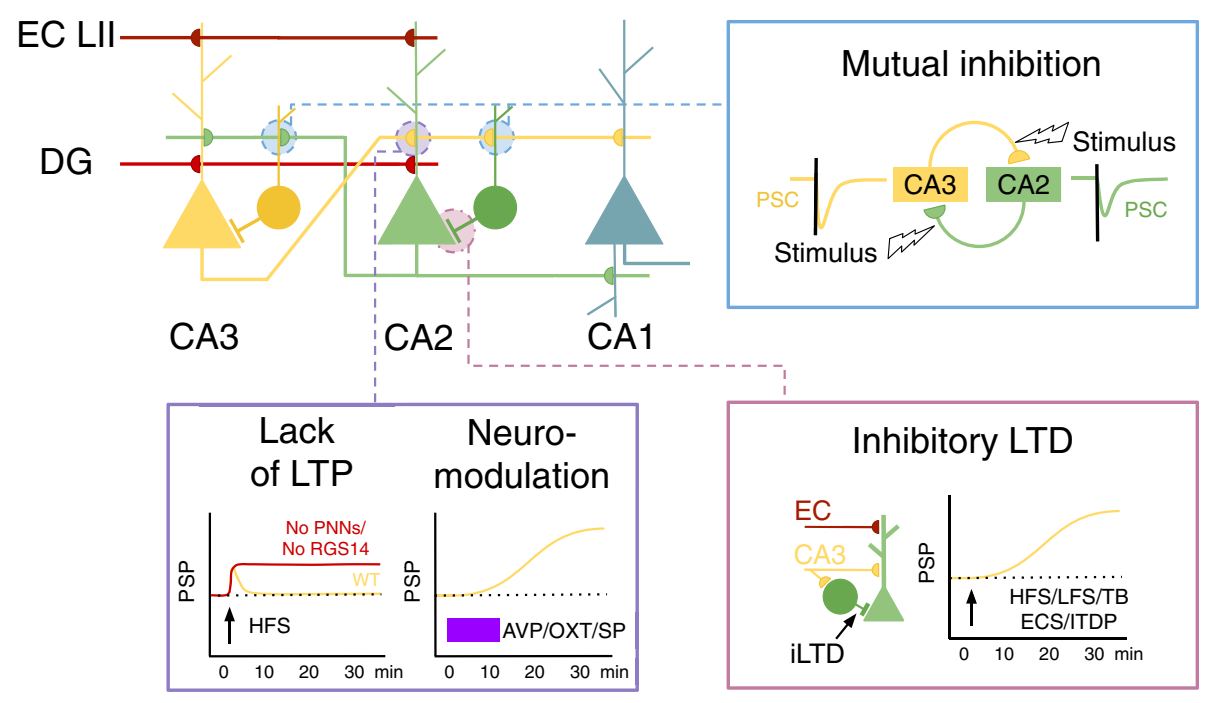

FIGURE 1 Interactions between CA3 and CA2 are characterized by mutual inhibition and restricted plasticity. Wiring diagram, upper left: In contrast to hippocampal region CA1, pyramidal cells in CA3 and CA2 receive input from entorhinal cortex layer II (ECL II) and the dentate gyrus (DG). Upper right box: Interactions between both regions are strongly dominated by feed-forward inhibition. Activating excitatory projections between CA3 and CA2 leads to a predominantly inhibitory response in the other region. Postsynaptic current (PSC). Lower left box: Excitatory projections from CA3 to CA2 are characterized by a lack of activity-induced long-term potentiation (yellow line). Long-term potentiation can be artificially unlocked by selectively blocking the expression of CA2 specific receptors like RGS14 or the removal of perineuronal nets, PNNs (red line). Alternatively, the release of the neuromodulators vasopressin, oxytocin or substance P leads to potentiation of exitatory projections (yellow line). Postsynaptic potential (PSP). Lower right box: Further, excitatory drive from CA3 to CA2 can be increased by reducing feed-forward inhibition via inhibitory long term depression (iLTD). iLTD can be induced by stimulating CA3 inputs with high (HFS), low frequency stimulation (LFS) or theta bursts (TB). Further, stimulating EC inputs (ECS) or precise timing of EC and CA3 inputs (ITDP), also leads to iLTD. In consequence the relative strength of feed-forward excitation from CA3 to CA2 increases. Note: Both potentiation of excitation and iLTD unfold slowly, peak after around 30 minutes and result in roughly a doubling of the postsynaptic potential 
Buzsaki, 1994), the DG (Kohara et al., 2014) and entorhinal cortex Layer II (Bartesaghi \& Gessi, 2004; Chevaleyre \& Siegelbaum, 2010; Kohara et al., 2014). Like CA3, axons of CA2 pyramidal cells widely project along the proximodistal (subdivided into CA1, CA2, and CA3a/ $\mathrm{b} / \mathrm{c}$ ) as well as the septotemporal axes. CA2 axons arborize within all CA regions (Li et al., 1994; Tamamaki, Abe, \& Nojyo, 1988), with some reaching into the DG (Ishizuka, Weber, \& Amaral, 1990). Pyramidal cells in dorsal CA2 have been shown to directly project to the ventral hippocampus (Meira et al., 2018; Okuyama, 2018; Tamamaki et al., 1988). Thus despite its small size, CA2 can integrate information from and exert influence over a large portion of the hippocampus.

Pyramidal cells in CA2 are recurrently connected. Within CA2, monosynaptic excitatory connections occur with a probability of around 1.4\%; seven identified connections in 502 tested pyramidal cell pairs (Okamoto \& Ikegaya, 2019). Recurrent excitatory connection probability in CA3 is $0.92 \%$ (Guzman, Schlögl, Frotscher, \& Jonas, 2016) and in CA1 0.6\% (Deuchars \& Thomson, 1996). Thus, experimental data suggest that recurrent excitatory connectivity in CA2 is more similar to CA3 (1.4 vs. $0.92 \%)$ than CA1 (1.4 vs. $0.6 \%)$. Interestingly, recurrent connections between pyramdidal cells inside CA2 appear to be spatially biased. Six out of the seven confirmed recurrent projections were oriented towards CA3 (Okamoto \& Ikegaya, 2019).

Zooming in along the proximodistal axis, it appears that CA2 and CA3a form a bidirectionally coupled network. Ishizuka et al. (1990) observed that axons of CA3 pyramidal cells branch more extensively in CA3a/b compared to CA3c. CA2 pyramidal cells project mostly to CA3a (Ishizuka et al., 1990; Tamamaki et al., 1988). In contrast to projections from CA3 to CA2, back-projections from CA2 to CA3 are thinner and sparser (Ishizuka et al., 1990). Further, the recurrent interactions between CA3 and CA2 are strictly controlled by high levels of feed-forward inhibition (Chevaleyre \& Siegelbaum, 2010; Kohara et al., 2014) and limited plasticity (Zhao, Choi, Obrietan, \& Dudek, 2007). Therefore, recurrent inhibition between CA2 and CA3 prohibit most spike propagation unless feed-forward excitation is either potentiated or inhibition reduced (Nasrallah et al., 2019; Nasrallah, Piskorowski, \& Chevaleyre, 2015).

\section{I NEUROMODULATION AND SYNAPTIC PLASTICITY IN CA2}

Excitatory projections from CA3 to CA2 do not express classical longterm potentiation (LTP; Zhao et al., 2007). This is due to strong calcium buffering (Simons, Escobedo, Yasuda, \& Dudek, 2009), plasticity limiting signalling pathways (Lee et al., 2010; Simons, Caruana, Zhao, \& Dudek, 2012), and dense perineuronal nets (Carstens, Phillips, PozzoMiller, Weinberg, \& Dudek, 2016, but see Domínguez et al., 2019). Various neuromodulatory inputs specifically converge on CA2 and modulate strictly controlled net excitation from CA3 (see Figure 1, lower two panels, for more details see Benoy, Dasgupta, and Sajikumar (2018)). It has been shown that plasticity of CA3 excitatory feed-forward projections can be unlocked by the release of vasopressin, oxytocin and substance $P$ in combination with synaptic activity (Dasgupta et al., 2017;
Pagani et al., 2015). In turn, net excitation from CA3 to CA2 can also be increased by long term depression of feed-forward inhibition (iLTD) (Piskorowski \& Chevaleyre, 2013; Nasrallah et al., 2015, 2019; Nasrallah, Piskorowski, \& Chevaleyre, 2016). iLTD is mediated by enkephalin, which acts via delta-opioid receptors (Piskorowski \& Chevaleyre, 2013). Multiple stimulation protocols at proximal (CA3) inputs allow iLTD induction, such as theta bursts and low or high frequency stimulation (Piskorowski \& Chevaleyre, 2013). Further, iLTD can be induced by stimulating distal (cortical) inputs (Nasrallah et al., 2016) or by precisely timing distal and proximal inputs, called input-timing-dependent plasticity (ITDP) (Leroy, Brann, Meira, \& Siegelbaum, 2017). Interestingly, plasticity induced by vasopressin, oxytocin, substance $P$, and enkephalin share similar dynamics. Net excitation increases slowly and peaks after around 30 minutes, roughly doubling the excitatory drive. [Correction added on 7 September, 2020, after first online publication: the duration in the previous sentence was changed from 20 to 30 minutes.]

While we have direct experimental evidence for plasticity inside CA3, we can only make assumptions about plasticity at projections from CA2 to CA3 and within CA2. Inside CA3, excitatory synapses exhibit symmetric spike-timing-dependent plasticity (Mishra, Kim, Guzman, \& Jonas, 2016) without requiring additional neuromodulation. Comparable to $\mathrm{CA} 3 \rightarrow \mathrm{CA} 2$ projections, feed-forward inhibition inside CA3 can be reduced by enkephalin-mediated iLTD (Domínguez et al., 2019; Leroy et al., 2017). To our knowledge no study has yet addressed plasticity of excitatory projections inside CA2 and from CA2 to CA3. However, it is known that axons from both regions arrive at similar locations as their recurrent counterparts (Ishizuka et al., 1990; Tamamaki et al., 1988). Therefore we assume that CA2 $\rightarrow$ CA3 projections are plastic. Net excitation may increase because of both potentiation of direct excitatory projections and iLTD at inhibitory feedforward projections. In contrast, due to the mentioned plasticitylimiting factors, recurrent excitatory projections inside CA2 likely do not express Hebbian-type long-term plasticity in their baseline mode.

\section{4 | FLEXIBLE SEQUENCE PRIORITIZATION IS REQUIRED FOR OPTIMIZED MEMORY CONSOLIDATION}

Both spatial and nonspatial tasks elicit temporal sequences of neuronal activation in the hippocampus, encoding consecutive aspects of a given experience (MacDonald, Lepage, Eden, \& Eichenbaum, 2011; Pastalkova, Itskov, Amarasingham, \& Buzsáki, 2008). Sequences play out on the behavioral, theta and sharp wave ripple timescale and may reflect either previous and current experiences or future expectations (Carey, Tanaka, \& van Der Meer, 2019; Diba \& Buzsáki, 2007; Foster \& Wilson, 2007; Gupta, Van Der Meer, Touretzky, \& Redish, 2010; O'Keefe, 1976; Olafsdottir, Barry, Saleem, Hassabis, \& Spiers, 2015; Singer, Carr, Karlsson, \& Frank, 2013; Wu, Haggerty, Kemere, \& Ji, 2017). We refer to co-activated cells as neuronal assemblies and to their respective sequences as assembly sequences. After an event, assembly sequences need to be reactivated to consolidate the respective experiences for long-term storage (Dupret, 
(a)

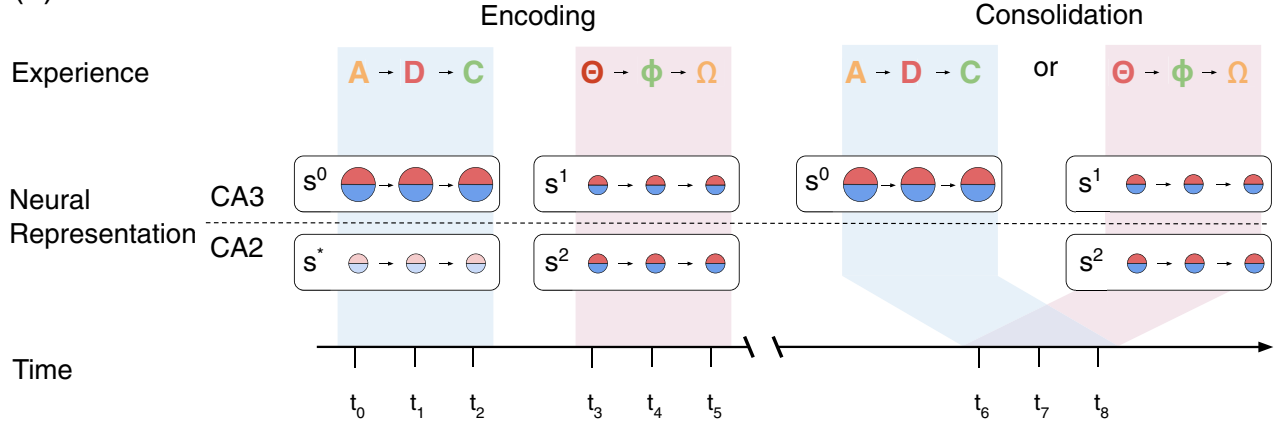

(b)

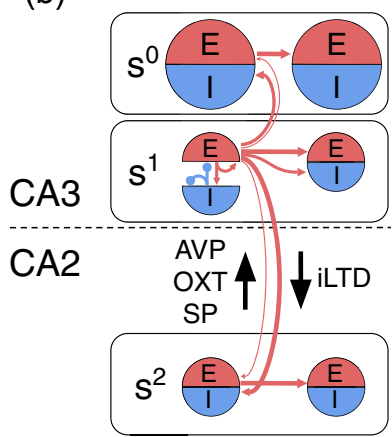

(c)

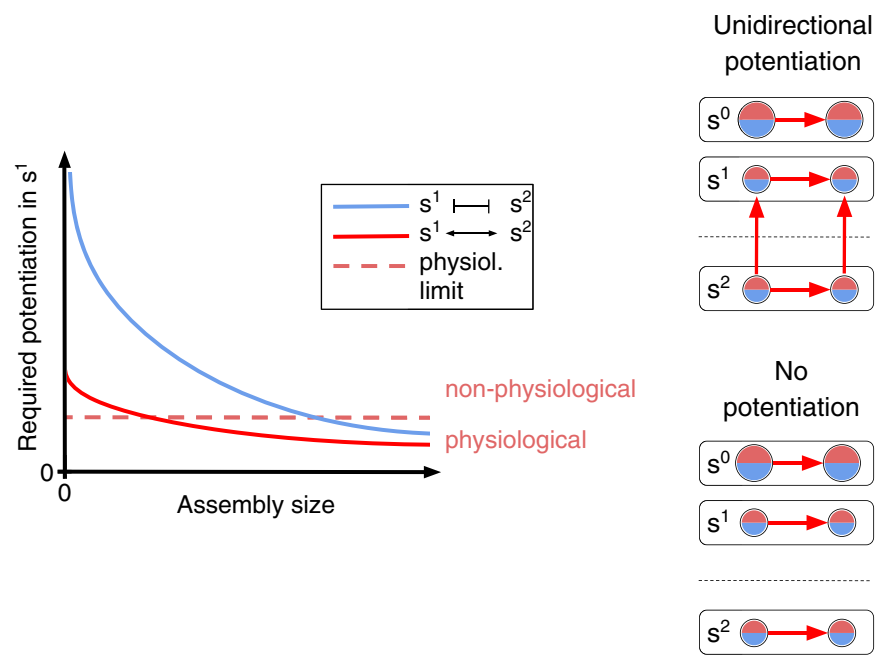

(d)

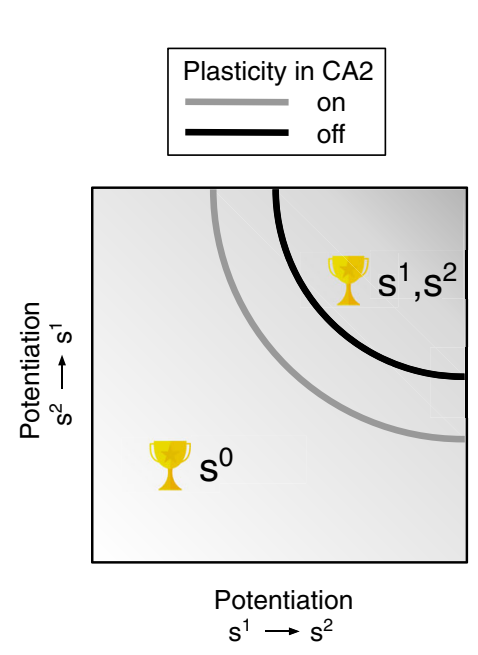

Bidirectional potentiation

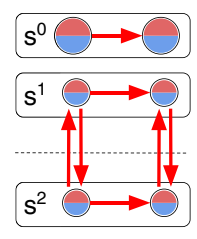

Unidirectional potentiation

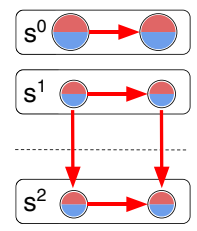

FIGURE 2 Interactions between CA3 and CA2 can define which sequences are replayed during consolidation. (a) Simplified scenario, two successive experiences elicit neural assembly sequences in CA3 and CA2 during encoding. During subsequent memory consolidation, the first assemblies of each sequence are simultaneously activated. Competition between assemblies leads to winner-take-all dynamics. First experience, $\mathrm{A} \rightarrow \mathrm{D} \rightarrow \mathrm{C}$, is represented by the strong CA3 sequence $s^{0}$. Sequential activity in CA2, $s^{*}$, is not paired and ignored. Second experience $\Theta \rightarrow \Phi \rightarrow \Omega$ is encoded as weak sequences in CA3 and CA2, $s^{1}$ and $s^{2}$. (b) Sequences are comprised of subsequently activated assemblies consisting of recurrently connected excitatory $(\mathrm{E})$ and inhibitory (I) neurons. Assemblies in strong sequence $s^{0}$ have more neurons (larger circles). Except for feed-forward excitation in all sequences, we show exemplary projections only for the first assembly of $s_{1}$. Feed-forward inhibition between CA3 and CA2 assemblies is particularly strong. For CA3 $\rightarrow$ CA2 projections, excitation can be conditionally increased by the release of vasopressin (AVP), oxytocin (OXT) and substance P (SP); while inhibition can be decreased by inhibitory long term depression (iLTD). (c) Dependence of required feed-forward potentiation on assembly size. We schematically illustrate the amount of required potentiation of feedforward weights in $s^{1}$ without (blue line, $s^{1} \longmapsto s^{2}$ ), and with mutual excitatory interactions with $s^{2}$ (red line, $s^{1} \leftrightarrow s^{2}$ ). (d) The graph in the middle shows how varying levels of potentiation between weak sequences $s^{1}$ and $s^{2}$ determine the outcome of the competition (indicated by the trophy). Without preferential interactions between $s^{1}$ and $s^{2}$, lower left corner, the strong sequence $s^{0}$ manages to inhibit $s^{1}$ and $s^{2}$. Unilateral potentiation between the weak sequences, upper left and lower right corner, is not sufficient to overcome the strong sequence. Only bidirectional potentiation, upper right corner, allows both weak sequences to support each others replay and to win over the strong sequence (gray line). If projections inside CA2 are not plastic, stronger sequence interactions can compensate for lack of feed-forward excitation (black line). For visual clarity, we show only projections with net excitation (red arrows)

O'Neill, Pleydell-Bouverie, \& Csicsvari, 2010; Fernández-Ruiz et al., 2019; Jadhav, Kemere, German, \& Frank, 2012; Singer et al., 2013). In their natural state, animals encounter a host of events and stimuli. Because animals do not form long-term memories of all events and stimuli, there must be mechanisms to prioritize which sequences should be replayed (Figure 2a).

Sequences representing different events/tasks likely deviate in the number of co-activated cells. In CA1, the position of other animals or inanimate objects is represented by smaller cell assemblies compared to an animal's own location (Danjo, Toyoizumi, \& Fujisawa, 2018; Omer, Maimon, Las, \& Ulanovsky, 2018). In addition, peak firing rates are lower for social compared to self place cells (Omer et al., 2018). Lower levels of activity may make it harder for an assembly to recruit further neurons and limit the amount of plasticity that can be induced during encoding.

A recent model by Chenkov, Sprekeler, and Kempter (2017) provides an intuitive understanding on how assembly sizes affect reactivation of individual sequences. For conceptual simplicity, assemblies 
are discrete populations of prewired, recurrently connected excitatory and inhibitory neurons. During an experience, external input is thought to activate assemblies in a temporal order, with co-activity leading to potentiation of feed-forward projections between subsequently activated assemblies. The necessary amount of feed-forward potentiation for successful reactivation depends nonlinearly on assembly sizes (Chenkov et al., 2017). A sequence of large assemblies requires little potentiation. In contrast, the amount of potentiation required for sequences with small assemblies may become unphysiologically large (Figure $2 \mathrm{c}$ ).

To understand sequence competition between multiple assembly sequences, we extend the model proposed by Chenkov et al. (2017). In its simplest form, competition between multiple sequences can be studied by considering only two sequences competing for reactivation in one network, here CA3. A strong sequence with large assemblies and a weak sequence with small assemblies. In particular, we assume that each sequence exerts feed-forward inhibition onto assemblies of competing sequences. In such a setting, if two sequences $s^{0}$ and $s^{1}$ are activated at the same time, for example by external input, the weaker sequence $s^{1}$ will disappear because the stronger sequence $s^{0}$ will recruit more inhibition onto the weaker sequence.

Each sequence, $s^{0}$ or $s^{1}$, is thought to represent an experience in one of two tasks, performed in close succession. For instance, $s^{0}$ may correspond to a classical spatial navigation task, for which we know that assemblies are relatively large, whereas $s^{1}$ may correspond to a task with small assemblies, like remembering the trajectory of another animal or an inanimate object. But, what if the experience of the weak sequence $s^{1}$ is much more relevant compared to the strong sequence $s^{0}$ ? Left as such, during subsequent consolidation, the strong sequence $s^{0}$ will be reactivated much more frequently than the weak sequence $s^{1}$.

How could the hippocampal circuit prioritize specific sequences despite different strengths? Such a prioritization of neuronal activity sequences is obviously important to form appropriate memories and an accurate model of the environment. Thus, we argue that there is a need for a sequence prioritization unit either within the hippocampus or in an upstream region. We propose that CA2 plays a role in strengthening sequences, increasing their chance to be replayed and thereby can perform the role of a sequence prioritization unit.

The framework we put forward is agnostic to content and timescale of sequences. To be prioritized during later reactivation, we only require that sequences, whatever they represent, are present during a neuromodulatory controlled pairing process. Therefore, we presuppose that CA2/CA3 have the necessary circuitry to generate sequences and focus on sequence competition and prioritization.

\section{5 | SEQUENCES IN CA2 AND CA3 MAY MUTUALLY SUPPORT EACH OTHER'S REACTIVATION}

In our sequence competition scenario, the weak sequence $s^{1}$ struggles for two reasons. Small assemblies require more feed-forward potentiation to sufficiently excite following assemblies. In addition, they recruit less feed-forward inhibition, making it more difficult to suppress competing sequences. In this situation, one can make use of the observation that sequential activity is also present in CA2 (Alexander et al., 2016; Lee, Wang, Deshmukh, \& Knierim, 2015; Mankin, Diehl, Sparks, Leutgeb, \& Leutgeb, 2015). Let $s^{2}$ be the CA2 sequence concurrently present with CA3 sequence $s^{1}$. Because assemblies in $s^{1}$ and $s^{2}$ are simultaneously active during encoding, and assuming coincidental neuromodulator release, they can team up and support each other via reciprocal potentiation. For the strong CA3 sequence $s^{0}$, we ignore the concurrently active CA2 sequence $s^{*}$ because we assume it was not associated with neuromodulatory release and hence not paired.

To understand how two sequences can support each other consider the schematic of $s^{0}, s^{1}, s^{2}$ shown in Figure 2b. Extending the computational model of sequence replay proposed by Chenkov et al. (2017) we can show that by increasing net excitation between $s^{1}$ and $s^{2}$, we can find conditions under which $s^{1}+s^{2}$ will overcome $s^{0}$ and replay more frequently (Figure $2 \mathrm{c}, \mathrm{d}$ ). This analysis suggests that a bidirectional increase in net excitation between $s^{1}$ and $s^{2}$ is necessary (Stöber et al., in prep.). It allows each side to benefit from additional feedback excitation while increasing inhibition on competing assemblies. Instead, if net excitation increases only in one direction, for example, from $s^{2}$ to $s^{1}$, increased feedback inhibition makes sequence reactivation more difficult.

Bidirectional excitatory interactions can compensate for the presumed lack of plasticity inside CA2. It remains unresolved whether recurrent CA2 projections are plastic. If not, assemblies may not be able to potentiate feed-forward projections, hindering the formation of assembly sequences inside CA2. By linking CA2 assemblies through CA3, reliable reactivation of CA2 sequences may nevertheless be possible. Increased net potentiation between CA2 and CA3 could compensate for the lack of feed-forward excitation in CA2 (Figure 2d, gray vs. black line).

In summary, the proposed sequence prioritization mechanism is based on a three-factor rule for synaptic potentiation, in at least one direction. Plasticity unfolds when three conditions are met: (a) pre-, and (b) postsynaptic activity, as well as (c) a salience signal. Mutual inhibition between the two networks helps suppress competing sequences and ensures that only correctly paired sequences in both networks are reactivated.

\section{6 | CA3-CA2 INTERACTIONS MAY IMPLEMENT SEQUENCE PRIORITIZATION}

Based on anatomical and physiological studies, we argue that the CA3-CA2 system is well suited to implement sequence prioritization via pairing of co-active sequences. Dense recurrent excitatory projections (Ishizuka, Cowan, \& Amaral, 1995; Kohara et al., 2014; Tamamaki et al., 1988) likely allow arbitrary cell assemblies to be linked within and across the two regions. Local inhibition within each region may create a winner-take-all scenario (Bazelot, Dinocourt, 
Cohen, \& Miles, 2010; Beyeler et al., 2013; Botcher, Falck, Thomson, \& Mercer, 2014). Strong reciprocal inhibition provides additional means for suppressing competing sequences.

Neuromodulatory-gated plasticity can selectively strengthen projections from CA3 to CA2. Under baseline conditions excitatory plasticity is strongly restricted (Carstens et al., 2016; Lee et al., 2010; Zhao et al., 2007). However, release of any of the neuromodulatory substances oxytocin, vasopressin or substance $P$ in combination with presynaptic activity leads to selective potentiation of activated excitatory synapses (Dasgupta et al., 2017; Pagani et al., 2015). In the case of vasopressin (Pagani et al., 2015) and substance P (Dasgupta et al., 2017) there is no effect, and in the case of oxytocin (Pagani et al., 2015), very little effect on synapses that are silent during the release. Furthermore, enkephalin-dependent iLTD selectively reduces feed-forward inhibition (Piskorowski \& Chevaleyre, 2013). iLTD may provide a complementary mechanism for sequence interactions and suggests that effective coupling relies on both potentiation of excitation and reduction of inhibition. In all cases, synaptic potentiation develops slowly. The slow onset may be important to avoid interference with encoding.

Neuromodulation in CA2 may act as salience cue. Neuromodulationgated plasticity in CA2 can arrive both from internal and external projections. iLTD depends on locally released enkephalin (Leroy et al., 2017). Vasopressin arrives via projections from the paraventricular nucleus (Smith et al., 2016; Swanson, Wyss, \& Cowan, 1978; Zhang \& Hernandez, 2013) and substance $P$ from the supramammiliary nucleus (Borhegyi \& Leranth, 1997; Cui, Gerfen, \& Young, 2013). There is strong evidence that the release of vasopressin and substance $P$ reflects experience of vital relevance to the animal. For example vasopressin is released in the dorsal hippocampus during parturition (Landgraf, Neumann, \& Pittman, 1991). Social recognition memory is blocked by the application of vasopressin anti-serum immediately after an encounter (van Wimersma Greidanus \& Maigret, 1996) and artificial release of vasopressin boosts the duration of social recognition memory (Smith et al., 2016). Further, vasopressin signaling is required for processing nonspatial sequence memories (DeVito et al., 2009). While the release of substance $P$ in the hippocampus has not been directly studied, the activity of its originating region, the supramamilliary nucleus, has been associated with environmental novelty (Ito, Shirao, Doya, \& Sekino, 2009), forced immobilization (Choi et al., 2012) and cold exposure (Miyata, Ishiyama, Shibata, Nakashima, \& Kiyohara, 1998). The latter two are stress situations that the animal likely wants to avoid in the future.

\section{7 | SEQUENCE PRIORITIZATION PROVIDES NEW INTERPRETATIONS OF EXPERIMENTAL FINDINGS}

We illustrate the sequence prioritization mechanisms in a very simplified scenario: With only three discretized and prewired assembly sequences. A strong, uncoupled assembly sequence in CA3 competes with two weak and mutually supportive sequences in CA3 and CA2. Only the latter two sequences receive neuromodulation and we ignore plasticity inside assemblies. All sequences are reactivated at the same time and only one sequence group can win, while the other is suppressed. In reality, the situation is obviously much more complicated. While awake, an animal is constantly experiencing, likely leading to a multitude of assembly sequences being activated in short time windows. And assembly sequences are not discrete but continuous. Assembly sizes may vary and even dynamically change over time. A complex cocktail of neuromodulators may be constantly present. During rest, internal dynamics may strongly influence network activity and reactivation may arise both spontaneously and upon external input. For those reasons, we expect that sequence reactivation is not a binary variable, but rather a probability distribution with multiple sequences, biased by the proposed sequence prioritization mechanism.

For a strong sequence, with many neurons participating at any given moment in time, potentiation of feed-forward synapses may suffice for successful propagation and inhibition of other competing sequences. In this case, help from CA2 may not be necessary. It has been shown that lesioning CA2 has no significant effect on spatial navigation in the Morris water maze (Hitti \& Siegelbaum, 2014). Since position is represented by a large number of pyramidal cells in the hippocampus, the respective memory traces may constitute strong sequences that do not require additional support. Yet, an interesting case arises when two such strong sequences compete. In the Morris water maze example, animals with CA2 lesions trended towards slower relearning of a new platform location (Hitti \& Siegelbaum, 2014). In our interpretation, behavioral sequences that reflect the new platform location are not sufficiently prioritized. Sequences reflecting the old location are still present during replay, slowing down the acquisition of the new location.

Increased activity in CA2 may compensate for fewer cells. To have significant effect on CA3, a CA2 sequence must recruit sufficient neuronal activity. CA2 may compensate for its disadvantage in size by letting cells participate in multiple cell assemblies. This argument is supported by CA2 place cells having multiple fields and being active across different environments (Lee et al., 2015; Lu, Igarashi, Witter, Moser, \& Moser, 2015; Mankin et al., 2015). The large spatial extent of CA2 place fields (Mankin et al., 2015; Oliva, Fernández-Ruiz, Buzsáki, \& Berényi, 2016b) may help bias the transition between CA3 assemblies.

Bidirectional sequence pairing allows combining diverse information in CA3 and CA2. Diverging place field properties (Lee et al., 2015; Lu et al., 2015; Mankin et al., 2015) indicate that CA3 and CA2 represent different information. In contrast to stable CA3 place fields, the CA2 population vector completely decorrelates on a timescale of hours (Mankin et al., 2015). With CA2/CA3a strongly responding to changes in local cues (Alexander et al., 2016; Lee et al., 2015; Wintzer, Boehringer, Polygalov, \& McHugh, 2014) and CA3b/c more prone to global cues (Lee et al., 2015; Mankin et al., 2015), one can speculate that the pairing of assembly sequences embeds variable local information into a stable global context. Both regions may then provide complementary information to downstream CA1.

In a related proposal, McHugh and colleagues suggest that a new CA2 subpopulation is recruited whenever sensory-based EC input differs sufficiently from memory-based CA3 input (Middleton \& McHugh, 2019; Wintzer et al., 2014). Such a situation would be of 
putative importance to the animal, and may therefore induce neuromodulator release. Within our framework, this would result in pairing of co-active CA2 and CA3 sequences and thus prioritization for replay. A new set of CA2 cells, and hence novel CA2 assembly sequences, would confer the additional benefit that the pairing not only prioritizes the salient experience for later replay, but also provides a unique index for the episode.

Two recently described subpopulations of CA2 pyramidal cells, N units (Kay et al., 2016) and ramping cells (Oliva et al., 2016a), fit nicely with our proposal of sequence prioritization. Despite some differences that require further clarification, it seems likely that these two terms are different descriptions of the same cell type. Ramping cells increase their firing rate before and are relatively silent during a sharp wave ripple event. $\mathrm{N}$ units are nonpositively modulated by sharp wave ripples, fire preferentially during immobility and are spatially selective. These properties may allow $\mathrm{N}$ units or ramping cells to bias sequence reactivation during sharp wave ripples by activating the first assemblies of a particular sequence. With more inhibition on other sequences and increased activation of subsequent assemblies, successful propagation of the selected sequence during the upcoming sharp wave ripple becomes more likely. As a consequence we expect that $\mathrm{N}$ units/ramping cells are co-activated in stable subgroups and that their activation predicts the replay of specific sequences (Middleton \& McHugh, 2019).

We expect that unconditionally unlocking plasticity of the CA3 $\rightarrow$ CA2 synapse will lead to general pairing of sequences in CA3 and CA2. Under such conditions, selective prioritization of important, yet weak sequences becomes difficult, because strong sequences may dominate even more. Unlocking plasticity can for example be achieved by selectively preventing the expression of plasticity limiting gene RGS14 (Lee et al., 2010). Consistent with our prediction, RGS14 ${ }^{-/}$ mice showed an increased learning rate in the Morris water maze and stronger responses in a novel object recognition task (Lee et al., 2010). However, we expect difficulties when multiple experiences with different relevance happen close in time. In such cases, prioritizing sequences during replay is integral for optimizing performance.

Artificially inducing net potentiation of the CA3 $\rightarrow$ CA2 synapse by releasing vasopressin, oxytocin, substance $P$ and/or induction of iLTD should prioritize replay of concurrently active CA2 and CA3 sequences. In contrast, deactivating CA2 during encoding or consolidation or preventing plasticity should disrupt prioritization. This can be tested for example in the object-trace-odor task. We expect that silencing CA2 will lead to a similar lack of temporal sequence memory as globally knocking out the Avpr1b receptor (DeVito et al., 2009).

Diverse actions of neuromodulation on cortical synapses (e.g., from EC LII, Chevaleyre \& Siegelbaum, 2010; Kohara et al., 2014) onto CA2 pyramidal cells can be interpreted in the light of sequence prioritization. In our simplified scenario, sequences are activated by external cortical synapses of equal strength. However, by modulating cortical synapses, one can influence the activity at the start of each sequence, thus providing an additional mechanism of sequence prioritization. For example, substance $\mathrm{P}$ potentiates cortical synapses active during its release (Dasgupta et al., 2017) and therefore may facilitate reactivation of the current experience. In contrast, vasopressin may hinder the reactivation of preceding experiences by selectively weakening previously potentiated synapses (Chafai, Corbani, Guillon, \& Desarménien, 2012).

Input-timing dependent plasticity weakens feed-forward inhibition between CA3 and CA2 and strengthens cortical projections (Leroy et al., 2017). As with substance $P$, stronger cortical projections may facilitate externally triggered reactivation. Whether ITDP is synapse specific and whether it allows linking individual cell assemblies remains to be resolved. In contrast to ITDP in CA1, ITDP in CA2 does not require postsynaptic activity. Further, ITDP seems to recruit at least two mediating interneuron subgroups (Leroy et al., 2017). Hence, we assume that ITDP is not specific and expect it to allow the recruitment of previously silent pyramidal cells during reactivation. Cells that were silent during encoding, but received matching cortical and CA3 input, will receive more net excitation during subsequent reactivation. Those cells likely recruit further inhibition in CA3, potentially blocking other competing sequences.

Alternative sequence prioritization mechanisms may exist besides the proposed CA3/CA2 sequence pairing. McNamara, Tejero-Cantero, Trouche, Campo-Urriza, and Dupret (2014) found that activating dopaminergic fibers in dorsal CA1 further increases the reinstatement of spatial firing patterns after exploring a novel environment. Thus, the study shows that spatial sequences can be prioritized locally in CA1. Since the link between dopamine release and increased reactivation remains elusive, at least two interpretations are possible. On the one hand, the dopamine-based mechanism could facilitate prioritization of local sequences inside CA1. Alternatively, since $\mathrm{CA} 1$ receives CA3 and CA2 input, dopamine signalling may determine the response strength to sequences arriving from the CA3/CA2 system (Rosen, Cheung, \& Siegelbaum, 2015). In the latter case, the dopamine-based mechanism would be additional to the CA3-CA2 prioritization. Further investigations are warranted.

\section{1 | Experiment to test the role of CA2 in sequence prioritization}

We outline one experiment to confirm or falsify our proposal that interactions between CA2 and CA3 allow one form of general sequence prioritization. The key is to have two tasks in close succession followed by a rest period to measure memory reactivation. In a very simple form, the tasks may entail running back and fourth on two different linear tracks (Diba \& Buzsáki, 2007). To avoid prioritization by other mechanisms outside of CA2 (McNamara et al., 2014), one could optogenetically excite neuromodulatory fibers exclusively inside CA2 in only one of the two tasks (Smith et al., 2016). These may be fibers releasing vasopressin, oxytocin or substance $P$. In subsequent rest, we expect that memory reactivation is biased towards the task during which CA2 mediated neuromodulation was released.

\section{2 | Predictions}

- CA2 plays a general role in episodic memory tasks, extending beyond social recognition memory. 
- Interplay between CA3 and CA2 selects which information is passed on to downstream CA1.

- Excitatory projections from CA2 to CA3 pyramidal cells are either plastic or prewired such that a subset expresses net-excitation.

- Inducing net potentiation at CA3-CA2 synapses prioritizes reactivation of concurrently active CA2 and CA3 sequences during later replay. [Correction added on 7 September, 2020, after first online publication: "replay" was changed to "reactivation" in this sentence.]

- Deactivating CA2 during encoding or consolidation, preventing or unconditionally unlocking plasticity should disrupt prioritization. In turn, selective release of neuromodulatory substances increases reactivation of concurrently activated sequences.

- Lack of sequence prioritization leads to behavioral deficits in complex environments, where important and nonimportant information needs to be distinguished.

\section{8 | DISCUSSION}

Investigations of CA2's functional role have only taken up pace in recent years. To our knowledge, our framework is the first attempt to build an overarching theory for CA2, integrating many of the fragmented anatomical and physiological insights. We propose that the recurrent CA3-CA2 system is, in the presence of a salience cue, able to prioritize sequences for replay. We assign a role to limited plasticity, selective neuromodulation and inhibitory plasticity at CA3-CA2 synapses.

The proposed framework is built on several strong assumptions. For example, we assumed that a three-factor learning rule underlies selective pairing of co-active assemblies, meaning that potentiation depends on pre- and postsynaptic activity as well as on neuromodulatory release. The influence of postsynaptic activity is yet to be tested for three of the mentioned neuromodulatory substances: vasopressin, oxytocin, and substance P (Dasgupta et al., 2017; Pagani et al., 2015). However, in case postsynaptic activity is not required, it is still conceivable that silent subpopulations in CA2 could be unidirectionally recruited by CA3 (see Leroy et al., 2017; Nasrallah et al., 2015), with bidirectional pairing taking place in a second step. Further, it is not known whether the insights from slice physiology transfer to the in vivo situation and under which behavioral conditions neuromodulatory substances are released. For conceptual simplicity, we assumed that assemblies are nonoverlapping and preconfigured. However, neither of these two properties are hard requirements. Similar dynamics underlie sequences with overlapping assemblies (Chenkov et al., 2017) and assembly formation and pairing could occur simultaneously.

Recent studies addressing CA2's relevance for memory have focused on social recognition memory. Recognition could simply rely on familiarity alone and thus may not depend on episodic memory (Brown \& Aggleton, 2001; Patai et al., 2015), making it independent of the mechanism we propose. However, our framework may also apply to social recognition memory for two reasons. First, episodic memories of another animal should strengthen its recognition. To our knowledge this has not been explicitly tested yet. Second, recently discovered place cells for others, with phase precession solely as a function of the other's location (Danjo et al., 2018; Omer et al., 2018), indicate that the hippocampus represents social information similar to other episodic memories (Buzsáki \& Tingley, 2018). For this reason we argue that mechanisms for prioritizing sequences for replay, also social sequences, should be of general nature.

We describe only the core mechanism of sequence prioritization. For conceptual simplicity we consider only two sets of sequences representing two distinct experiences, but a similar winner-take-all mechanisms ought to work for more than two sets of sequences, given enough recurrent excitation to recover from initial feed-forward inhibition. So far, it is not clear whether the different neuromodulators act together and what specific role they play. Experimental evidence suggests they complement each other. For example, social recognition memory depends on vasopressin (Wersinger et al., 2002), oxytocin (Lin et al., 2018; Raam, McAvoy, Besnard, Veenema, \& Sahay, 2017) and enkephalin (Leroy et al., 2017). In any case, enkephalin-mediated iLTD appears to be a special case. It is the only mechanism for which a) the neuromodulator releasing cells are in close proximity and b) it is not necessary to add enkephalin to the acute slice experiments to unlock plasticity (Leroy et al., 2017; Piskorowski \& Chevaleyre, 2013). It is therefore conceivable that iLTD is active in the baseline mode and the other neuromodulatory substances work on top of it.

Separating sequence generation (within CA2 and CA3) from sequence control/prioritization (CA2 $\leftrightarrow$ CA3 projections) confers a number of advantages. As mentioned above, beyond prioritization, pairing of CA2 and CA3 assembly sequences could provide a unique index for novel/salient episodes and embed local information into global contexts. In addition, it could also tease apart overlapping CA3 sequences. For example, if two CA3 sequences with overlapping assemblies (e.g., $\mathrm{A} \rightarrow \mathrm{B} \rightarrow \mathrm{C}$ vs. $\mathrm{A} \rightarrow \mathrm{C} \rightarrow \mathrm{D}$ ) receive input to the first assembly (A), the sequence with the strongest feed-forward projections and largest assemblies will win out, the other being suppressed. In this case, it comes down to the relative strength of projections from $A \rightarrow B$ vs. $A \rightarrow C$. However, if the CA3 sequences are paired with two different $\mathrm{CA} 2$ sequences ( $\mathrm{U} \rightarrow \mathrm{V} \rightarrow \mathrm{W}$ and $\mathrm{X} \rightarrow \mathrm{Y} \rightarrow \mathrm{Z}$, respectively) then activation of assembly $A$ in $C A 3$ and either $U$ or $X$ from the paired CA2 sequence determines how replay will progress. Given that $C A 2$ activity reorganizes after changes in local cues (Alexander et al., 2016; Lee et al., 2015; Wintzer et al., 2014) and decorrelates on a timescale of hours (Mankin et al., 2015), new CA2 sequences could be readily made available to pair with, and hence prioritize, stable CA3 sequences. These pairings could then influence replay and thus memory consolidation primarily in the first hours after an experience, after which the recruited neurons in CA2 may be flexibly reused to form new CA2-CA3 pairings.

\section{9 | CONCLUSION}

We propose that the hippocampus prioritizes important neural activity sequences, increasing the probability of their subsequent replay. We have formulated a conceptual framework that allows the CA3- 
CA2 system to control which sequences are reactivated. Namely, neuromodulatory-gated plasticity and mutual inhibition enable sequences in both regions to support each other while suppressing competing sequences. In conclusion, considering CA2 as a sequence prioritization unit provides a cohesive interpretation of its unique functional properties and makes the first steps towards incorporating CA2 into an overarching theory of hippocampal memory processing.

\section{ACKNOWLEDGMENTS}

The authors thank Sen Cheng, Serena Dudek, Thomas McHugh, Steven Siegelbaum, Rebecca Piskorowski, Felix Leroy, Antonio FernándezRuiz, Azahara Oliva, Scott H. Deibel, Trygve Solstad and Ane Charlotte Christensen for insightful discussions leading to the development of this framework. This work was supported by Research Council of Norway's Grant No. 250259 and 248828 to Marianne Fyhn as well as the University of Oslo (Marianne Fyhn, Tristan M. Stöber, Torkel Hafting, Andrew B. Lehr) and Simula Research Laboratory (Tristan M. Stöber, Andrew B. Lehr). AK is supported by the Swedish Research Council: Research Project Grant and Strategic Research Area: StratNeuro. Tristan M. Stöber (PhD fellow) and Marianne Fyhn (PI) are part of the Simula-UCSD-University of Oslo Research and PhD training (SUURPh) program, an international collaboration in computational biology and medicine funded by the Norwegian Ministry of Education and Research. Further, we thank the LifeScience internationalization grant by the University of Oslo for supporting the collaboration with KTH Royal Institute of Technology in Stockholm.

\section{DATA AVAILABILITY STATEMENT}

Data sharing is not applicable to this article as no new data were created or analyzed in this study.

\section{ORCID}

\section{Tristan M. Stöber (D) https://orcid.org/0000-0003-3853-0608}

\section{REFERENCES}

Alexander, G. M., Brown, L. Y., Farris, S., Lustberg, D., Pantazis, C., Gloss, B., ... Dudek, S. M. (2018). CA2 neuronal activity controls hippocampal low gamma and ripple oscillations. eLife, 7, e38052.

Alexander, G. M., Farris, S., Pirone, J. R., Zheng, C., Colgin, L. L., \& Dudek, S. M. (2016). Social and novel contexts modify hippocampal CA2 representations of space. Nature Communications, 7(1), 1-14.

Bartesaghi, R., \& Gessi, T. (2004). Parallel activation of field CA2 and dentate gyrus by synaptically elicited perforant path volleys. Hippocampus, 14(8), 948-963.

Bazelot, M., Dinocourt, C., Cohen, I., \& Miles, R. (2010). Unitary inhibitory field potentials in the CA3 region of rat hippocampus. The Journal of Physiology, 588(12), 2077-2090.

Benoy, A., Dasgupta, A., \& Sajikumar, S. (2018). Hippocampal area CA2: An emerging modulatory gateway in the hippocampal circuit. Experimental Brain Research, 236(4), 919-931.

Beyeler, A., Retailleau, A., Molter, C., Mehidi, A., Szabadics, J., \& Leinekugel, X. (2013). Recruitment of perisomatic inhibition during spontaneous hippocampal activity in vitro. PLoS One, 8(6), e66509.

Boehringer, R., Polygalov, D., Huang, A. J., Middleton, S. J., Robert, V., Wintzer, M. E., ... McHugh, T. J. (2017). Chronic loss of CA2 transmission leads to hippocampal hyperexcitability. Neuron, 94(3), 642-655.
Borhegyi, Z., \& Leranth, C. (1997). Substance P innervation of the rat hippocampal formation. Journal of Comparative Neurology, 384(1), 41-58.

Botcher, N. A., Falck, J. E., Thomson, A. M., \& Mercer, A. (2014). Distribution of interneurons in the CA2 region of the rat hippocampus. Frontiers in Neuroanatomy, 8, 104.

Brown, M. W., \& Aggleton, J. P. (2001). Recognition memory: What are the roles of the perirhinal cortex and hippocampus? Nature Reviews Neuroscience, 2(1), 51-61.

Buzsáki, G., \& Tingley, D. (2018). Space and time: The hippocampus as a sequence generator. Trends in Cognitive Sciences, 22(10), 853-869.

Carey, A. A., Tanaka, Y., \& van Der Meer, M. A. (2019). Reward revaluation biases hippocampal replay content away from the preferred outcome. Nature Neuroscience, 22, 1450-1459.

Carstens, K. E., Phillips, M. L., Pozzo-Miller, L., Weinberg, R. J., \& Dudek, S. M. (2016). Perineuronal nets suppress plasticity of excitatory synapses on CA2 pyramidal neurons. The Journal of Neuroscience, 36(23), 6312-6320.

Chafai, M., Corbani, M., Guillon, G., \& Desarménien, M. G. (2012). Vasopressin inhibits LTP in the CA2 mouse hippocampal area. PLoS One, 7 (12), e49708.

Chenkov, N., Sprekeler, H., \& Kempter, R. (2017). Memory replay in balanced recurrent networks. PLoS Computational Biology, 13(1), e1005359.

Chevaleyre, V., \& Siegelbaum, S. A. (2010). Strong CA2 pyramidal neuron synapses define a powerful disynaptic cortico-hippocampal loop. Neuron, 66(4), 560-572.

Choi, W. K., Wirtshafter, D., Park, H.-J., Lee, M.-S., Her, S., \& Shim, I. (2012). The characteristics of supramammillary cells projecting to the hippocampus in stress response in the rat. The Korean Journal of Physiology \& Pharmacology, 16(1), 17-24.

Cui, Z., Gerfen, C. R., \& Young, W. S. (2013). Hypothalamic and other connections with dorsal CA2 area of the mouse hippocampus. Journal of Comparative Neurology, 521(8), 1844-1866.

Danjo, T., Toyoizumi, T., \& Fujisawa, S. (2018). Spatial representations of self and other in the hippocampus. Science, 359(6372), 213-218.

Dasgupta, A., Baby, N., Krishna, K., Hakim, M., Wong, Y. P., Behnisch, T., ... Sajikumar, S. (2017). Substance $P$ induces plasticity and synaptic tagging/capture in rat hippocampal area CA2. Proceedings of the National Academy of Sciences, 114(41), E8741-E8749.

Deuchars, J., \& Thomson, A. (1996). CA1 pyramid-pyramid connections in rat hippocampus in vitro: Dual intracellular recordings with biocytin filling. Neuroscience, 74(4), 1009-1018.

DeVito, L. M., Konigsberg, R., Lykken, C., Sauvage, M., Young, W. S., \& Eichenbaum, H. (2009). Vasopressin $1 \mathrm{~b}$ receptor knock-out impairs memory for temporal order. The Journal of Neuroscience, 29(9), 2676-2683.

Diba, K., \& Buzsáki, G. (2007). Forward and reverse hippocampal place-cell sequences during ripples. Nature Neuroscience, 10(10), 1241-1242.

Domínguez, S., Rey, C. C., Therreau, L., Fanton, A., Massotte, D., Verret, L., ... Chevaleyre, V. (2019). Maturation of PNN and ErbB4 signaling in area CA2 during adolescence underlies the emergence of PV interneuron plasticity and social memory. Cell Reports, 29(5), 1099-1112.

Dupret, D., O'Neill, J., Pleydell-Bouverie, B., \& Csicsvari, J. (2010). The reorganization and reactivation of hippocampal maps predict spatial memory performance. Nature Neuroscience, 13(8), 995-1002.

Fernández-Ruiz, A., Oliva, A., de Oliveira, E. F., Rocha-Almeida, F., Tingley, D., \& Buzsáki, G. (2019). Long-duration hippocampal sharp wave ripples improve memory. Science, 364(6445), 1082-1086.

Foster, D. J., \& Wilson, M. A. (2007). Hippocampal theta sequences. Hippocampus, 17(11), 1093-1099.

Gluck, M. A., \& Rumelhart, D. E. (1990). Neuroscience and Connectionist Theory (Vol. 1, Hillsdale, New Jersey). Lawrence Erlbaum Associates.

Gupta, A. S., Van Der Meer, M. A. A., Touretzky, D. S., \& Redish, A. D. (2010). Hippocampal replay is not a simple function of experience. Neuron, 65(5), 695-705. 
Guzman, S. J., Schlögl, A., Frotscher, M., \& Jonas, P. (2016). Synaptic mechanisms of pattern completion in the hippocampal CA3 network. Science, 353(6304), 1117-1123.

Hitti, F. L., \& Siegelbaum, S. A. (2014). The hippocampal CA2 region is essential for social memory. Nature, 508(7494), 88-92.

Ishizuka, N., Cowan, W. M., \& Amaral, D. G. (1995). A quantitative analysis of the dendritic organization of pyramidal cells in the rat hippocampus. Journal of Comparative Neurology, 362(1), 17-45.

Ishizuka, N., Weber, J., \& Amaral, D. G. (1990). Organization of intrahippocampal projections originating from CA3 pyramidal cells in the rat. Journal of Comparative Neurology, 295(4), 580-623.

Ito, M., Shirao, T., Doya, K., \& Sekino, Y. (2009). Three-dimensional distribution of fos-positive neurons in the supramammillary nucleus of the rat exposed to novel environment. Neuroscience Research, 64(4), 397-402.

Jadhav, S. P., Kemere, C., German, P. W., \& Frank, L. M. (2012). Awake hippocampal sharp-wave ripples support spatial memory. Science, 336 (6087), 1454-1458.

Kay, K., Sosa, M., Chung, J. E., Karlsson, M. P., Larkin, M. C., \& Frank, L. M. (2016). A hippocampal network for spatial coding during immobility and sleep. Nature, 531(7593), 185-190.

Kohara, K., Pignatelli, M., Rivest, A. J., Jung, H.-Y., Kitamura, T., Suh, J., ... Tonegawa, S. (2014). Cell type-specific genetic and optogenetic tools reveal hippocampal CA2 circuits. Nature Neuroscience, 17(2), 269-279.

Landgraf, R., Neumann, I., \& Pittman, Q. J. (1991). Septal and hippocampal release of vasopressin and oxytocin during late pregnancy and parturition in the rat. Neuroendocrinology, 54(4), 378-383.

Lee, H., Wang, C., Deshmukh, S., \& Knierim, J. (2015). Neural population evidence of functional heterogeneity along the CA3 transverse axis: Pattern completion versus pattern separation. Neuron, 87(5), 1093-1105.

Lee, S. E., Simons, S. B., Heldt, S. A., Zhao, M., Schroeder, J. P., Vellano, C. P., ... Hepler, J. R. (2010). RGS14 is a natural suppressor of both synaptic plasticity in CA2 neurons and hippocampal-based learning and memory. Proceedings of the National Academy of Sciences, 107 (39), 16994-16998.

Leroy, F., Brann, D. H., Meira, T., \& Siegelbaum, S. A. (2017). Input-timingdependent plasticity in the hippocampal CA2 region and its potential role in social memory. Neuron, 95(5), 1089-1102.

Leutgeb, J. K., Leutgeb, S., Moser, M.-B., \& Moser, E. I. (2007). Pattern separation in the dentate gyrus and CA3 of the hippocampus. Science, 315(5814), 961-966.

Li, X.-G., Somogyi, P., Ylinen, A., \& Buzsáki, G. (1994). The hippocampal CA3 network: An in vivo intracellular labeling study. Journal of Comparative Neurology, 339(2), 181-208.

Lin, Y.-T., Hsieh, T.-Y., Tsai, T.-C., Chen, C.-C., Huang, C.-C., \& Hsu, K.-S. (2018). Conditional deletion of hippocampal CA2/CA3a oxytocin receptors impairs the persistence of long-term social recognition memory in mice. Journal of Neuroscience, 38(5), 1218-1231.

Lu, L., Igarashi, K. M., Witter, M. P., Moser, E. I., \& Moser, M.-B. (2015). Topography of place maps along the CA3-to-CA2 axis of the hippocampus. Neuron, 87(5), 1078-1092.

MacDonald, C. J., Lepage, K. Q., Eden, U. T., \& Eichenbaum, H. (2011). Hippocampal "time cells" bridge the gap in memory for discontiguous events. Neuron, 71(4), 737-749.

Mankin, E. A., Diehl, G. W., Sparks, F. T., Leutgeb, S., \& Leutgeb, J. K. (2015). Hippocampal CA2 activity patterns change over time to a larger extent than between spatial contexts. Neuron, 85(1), 190-201.

Marr, D. (1971). Simple memory: A theory for archicortex. Philosophical Transactions of the Royal Society of London B: Biological Sciences, 262, 23-81.

McNamara, C. G., Tejero-Cantero, Á., Trouche, S., Campo-Urriza, N., \& Dupret, D. (2014). Dopaminergic neurons promote hippocampal reactivation and spatial memory persistence. Nature Neuroscience, 17(12), 1658-1660.
Meira, T., Leroy, F., Buss, E. W., Oliva, A., Park, J., \& Siegelbaum, S. A. (2018). A hippocampal circuit linking dorsal CA2 to ventral CA1 critical for social memory dynamics. Nature Communications, 9(1), 4163.

Middleton, S. J., \& McHugh, T. J. (2019). CA2: A highly connected intrahippocampal relay. Annual Review of Neuroscience, 43, 2020.

Mishra, R. K., Kim, S., Guzman, S. J., \& Jonas, P. (2016). Symmetric spike timing-dependent plasticity at CA3-CA3 synapses optimizes storage and recall in autoassociative networks. Nature Communications, 7 (1), 1-11.

Miyata, S., Ishiyama, M., Shibata, M., Nakashima, T., \& Kiyohara, T. (1998). Infant cold exposure changes Fos expression to acute cold stimulation in adult hypothalamic brain regions. Neuroscience Research, 31(3), 219-225.

Nasrallah, K., Piskorowski, R. A., \& Chevaleyre, V. (2015). Inhibitory plasticity permits the recruitment of CA2 pyramidal neurons by CA3. eNeuro, 2(4), ENEURO.0049.

Nasrallah, K., Piskorowski, R. A., \& Chevaleyre, V. (2016). Bi-directional interplay between proximal and distal inputs to CA2 pyramidal neurons. Neurobiology of Learning and Memory, 138, 173-181.

Nasrallah, K., Therreau, L., Robert, V., Huang, A. J., McHugh, T. J., Piskorowski, R. A., \& Chevaleyre, V. (2019). Routing hippocampal information flow through parvalbumin interneuron plasticity in area CA2. Cell Reports, 27(1), 86-98.

O'Keefe, J. (1976). Place units in the hippocampus of the freely moving rat. Experimental Neurology, 51(1), 78-109.

Okamoto, K., \& Ikegaya, Y. (2019). Recurrent connections between CA2 pyramidal cells. Hippocampus, 29(4), 305-312.

Okuyama, T. (2018). Social memory engram in the hippocampus. Neuroscience Research, 129, 17-23.

Okuyama, T., Kitamura, T., Roy, D. S., Itohara, S., \& Tonegawa, S. (2016). Ventral CA1 neurons store social memory. Science, 353(6307), 1536-1541.

Olafsdottir, H. F., Barry, C., Saleem, A. B., Hassabis, D., \& Spiers, H. J. (2015). Hippocampal place cells construct reward related sequences through unexplored space. elife, 4, e06063.

Oliva, A., Fernández-Ruiz, A., Buzsáki, G., \& Berényi, A. (2016a). Role of hippocampal CA2 region in triggering sharp-wave ripples. Neuron, 91 (6), 1342-1355.

Oliva, A., Fernández-Ruiz, A., Buzsáki, G., \& Berényi, A. (2016b). Spatial coding and physiological properties of hippocampal neurons in the cornu ammonis subregions. Hippocampus, 26(12), 1593-1607.

Omer, D. B., Maimon, S. R., Las, L., \& Ulanovsky, N. (2018). Social placecells in the bat hippocampus. Science, 359(6372), 218-224.

Pagani, J. H., Zhao, M., Cui, Z., Avram, S. W., Caruana, D. A., Dudek, S. M., \& Young, W. (2015). Role of the vasopressin 1b receptor in rodent aggressive behavior and synaptic plasticity in hippocampal area CA2. Molecular Psychiatry, 20(4), 490-499.

Papp, G., Witter, M. P., \& Treves, A. (2007). The CA3 network as a memory store for spatial representations. Learning \& Memory, 14(11), 732-744.

Pastalkova, E., Itskov, V., Amarasingham, A., \& Buzsáki, G. (2008). Internally generated cell assembly sequences in the rat hippocampus. Science, 321(5894), 1322-1327.

Patai, E. Z., Gadian, D. G., Cooper, J. M., Dzieciol, A. M., Mishkin, M., \& Vargha-Khadem, F. (2015). Extent of hippocampal atrophy predicts degree of deficit in recall. Proceedings of the National Academy of Sciences, 112(41), 12830-12833.

Piskorowski, R. A., \& Chevaleyre, V. (2013). Delta-opioid receptors mediate unique plasticity onto parvalbumin-expressing interneurons in area CA2 of the hippocampus. The Journal of Neuroscience, 33(36), 14567-14578.

Raam, T., McAvoy, K. M., Besnard, A., Veenema, A., \& Sahay, A. (2017). Hippocampal oxytocin receptors are necessary for discrimination of social stimuli. Nature Communications, 8(1), 2001.

Rolls, E. T., \& Treves, A. (1998). Neural networks and brain function (Vol. 572). Oxford: Oxford University Press. 
Rosen, Z. B., Cheung, S., \& Siegelbaum, S. A. (2015). Midbrain dopamine neurons bidirectionally regulate CA3-CA1 synaptic drive. Nature Neuroscience, 18(12), 1763-1771.

Simons, S. B., Caruana, D. A., Zhao, M., \& Dudek, S. M. (2012). Caffeineinduced synaptic potentiation in hippocampal CA2 neurons. Nature Neuroscience, 15(1), 23-25.

Simons, S. B., Escobedo, Y., Yasuda, R., \& Dudek, S. M. (2009). Regional differences in hippocampal calcium handling provide a cellular mechanism for limiting plasticity. Proceedings of the National Academy of Sciences, 106(33), 14080-14084.

Singer, A. C., Carr, M. F., Karlsson, M. P., \& Frank, L. M. (2013). Hippocampal swr activity predicts correct decisions during the initial learning of an alternation task. Neuron, 77(6), 1163-1173.

Smith, A., Avram, S. W., Cymerblit-Sabba, A., Song, J., \& Young, W. (2016). Targeted activation of the hippocampal CA2 area strongly enhances social memory. Molecular Psychiatry, 21(8), 1137-1144.

Stevenson, E. L., \& Caldwell, H. K. (2014). Lesions to the CA2 region of the hippocampus impair social memory in mice. European Journal of Neuroscience, 40(9), 3294-3301.

Swanson, L., Wyss, J., \& Cowan, W. (1978). An autoradiographic study of the organization of intrahippocampal association pathways in the rat Journal of Comparative Neurology, 181(4), 681-715.

Tamamaki, N., Abe, K., \& Nojyo, Y. (1988). Three-dimensional analysis of the whole axonal arbors originating from single CA2 pyramidal neurons in the rat hippocampus with the aid of a computer graphic technique. Brain Research, 452(1), 255-272.

Treves, A., \& Rolls, E. T. (1992). Computational constraints suggest the need for two distinct input systems to the hippocampal CA3 network. Hippocampus, 2(2), 189-199.

van Wimersma Greidanus, T. B., \& Maigret, C. (1996). The role of limbic vasopressin and oxytocin in social recognition. Brain Research, 713(1), 153-159.

Wersinger, S., Ginns, E. I., O'carroll, A., Lolait, S., \& Young lii, W. (2002). Vasopressin $\mathrm{v} 1 \mathrm{~b}$ receptor knockout reduces aggressive behavior in male mice. Molecular Psychiatry, 7(9), 975-984.

Wersinger, S. R., Caldwell, H. K., Christiansen, M., \& Young, W. S. (2007). Disruption of the vasopressin $1 \mathrm{~b}$ receptor gene impairs the attack component of aggressive behavior in mice. Genes, Brain and Behavior, 6(7), 653-660.

Wersinger, S. R., Kelliher, K. R., Zufall, F., Lolait, S. J., O'Carroll, A.-M., \& Young, W. S. (2004). Social motivation is reduced in vasopressin $1 \mathrm{~b}$ receptor null mice despite normal performance in an olfactory discrimination task. Hormones and Behavior, 46(5), 638-645.

Wersinger, S. R., Temple, J. L., Caldwell, H. K., \& Young, W. S., 3rd. (2008). Disruption of the vasopressin $1 \mathrm{~b}$ receptor gene impairs the attack component of aggressive behavior in mice. Endocrinology, 149(1), 116-121.

Wintzer, M. E., Boehringer, R., Polygalov, D., \& McHugh, T. J. (2014). The hippocampal CA2 ensemble is sensitive to contextual change. The Journal of Neuroscience, 34(8), 3056-3066.

Wu, C.-T., Haggerty, D., Kemere, C., \& Ji, D. (2017). Hippocampal awake replay in fear memory retrieval. Nature Neuroscience, 20(4), 571-580.

Young, W. S., Li, J., Wersinger, S. R., \& Palkovits, M. (2006). The vasopres$\sin 1 \mathrm{~b}$ receptor is prominent in the hippocampal area CA2 where it is unaffected by restraint stress or adrenalectomy. Neuroscience, 143(4), 1031-1039.

Zhang, L., \& Hernandez, V. (2013). Synaptic innervation to rat hippocampus by vasopressin-immuno-positive fibres from the hypothalamic supraoptic and paraventricular nuclei. Neuroscience, 228, 139-162.

Zhao, M., Choi, Y.-S., Obrietan, K., \& Dudek, S. M. (2007). Synaptic plasticity (and the lack thereof) in hippocampal CA2 neurons. The Journal of Neuroscience, 27(44), 12025-12032.

How to cite this article: Stöber TM, Lehr AB, Hafting T, Kumar A, Fyhn M. Selective neuromodulation and mutual inhibition within the CA3-CA2 system can prioritize sequences for replay. Hippocampus. 2020;30:1228-1238. https://doi.org/10.1002/hipo.23256 